\title{
Sea Ice Remote Sensing Using Surface Reflected GPS Signals
}

\author{
Attila Komjathy', James Maslanik', Valery U. Zavorotny', Penina Axelrad' and Stephen J. Katzberg ${ }^{3}$ \\ 'CCAR/University of Colorado, CB 431, Boulder, CO 80309 \\ ${ }^{2}$ NOAA/Environmental Technology Laboratory, 325 Broadway, Boulder, CO 80303 \\ ${ }^{3}$ NASA Langley Research Center, Code 328, Hampton, VA, 23681-0001 \\ Phone: (303) 492-4829; Fax: (303)-492-2825; Email: komjathy@colorado.edu
}

\begin{abstract}
This paper describes a new research effort to extend the application of Global Positioning System (GPS) signal reflections, received by airborne instruments, to cryospheric remote sensing. Our experimental results indicate that reflected GPS signals have potential to provide information on the presence and condition of sea and freshwater ice as well as the freeze/thaw state of frozen ground. In this paper we show results from aircraft experiments over the ice pack near Barrow, Alaska indicating correlation between forward-scattered GPS returns and RADARSAT backscattered measurements.
\end{abstract}

\section{INTRODUCTION}

In 1996, Drs. Katzberg and Garrison of NASA Langley Research Center (LaRC) developed the idea of using reflected GPS signals for remote sensing applications, and published a number of papers describing the theory and mechanisms for this technique [e.g., 1-3]. Since then, research has advanced our understanding of reflected GPS signals and experimentally applied those techniques to ocean remote sensing and mapping [4-10]. The current investigation extends this work into cryospheric applications of GPS reflections based upon a proposal made by Drs. Katzberg and Garrison, to collect GPS signals reflected by ice surfaces. Results are shown from the first GPS measurements from sca ice in the Beaufort Sea in April 1998.

\section{MEASUREMENT METHODS}

The use of GPS in a bistatic radar configuration to measure surface properties relies upon our ability to extract information from the reflected signal. For standard GPS navigation purposes, the GPS receiver measures the signal delay from the satellite (the pseudorange measurement) and the rate of change of the range (the Doppler measurement). For remote sensing applications, the primary measurement is the received power from the reflected signal for a number of delays and Doppler values. This measurement and its sensitivity to the surface conditions are discussed in the following paragraphs.

The GPS satellites transmit two right-hand circularly polarized (RHCP) L-band signals at $1.57542 \mathrm{GHz}$ and 1.2276 GHz. These carrier signals are modulated by unique pseudorandom noise (PRN) codes with an autocorrelation function similar to the ideal autocorrelation function. The autocorrelation power has a triangular shape, which is suppressed at delays of more than \pm 1 chip $(300 \mathrm{~m}$ for the civilian (C/A) code). To acquire and track a signal, a conventional GPS receiver generates a local replica of the code for the particular satellite signal to be tracked, compensating for the expected Doppler shift and computing the cross-correlation between the replica and the incoming codes.

When a GPS signal encounters an ideal, smooth reflecting surface, specular reflection occurs at a single point. The reflected signal code structure remains, but the polarization of the wave is reversed to left-hand circular polarization (LHCP), and the signal power is decreased. If, however, the surface is rough relative to the GPS wavelength of $19 \mathrm{~cm}$, reflections are produced by multiple facets on the surface. Around the ideal specular point, this creates a so-called glistening zone within which there is a distribution of varying ranges and Doppler shifts, as shown in Fig. 1. To measure GPS signals reflected from land and sea surfaces, Garrison and Katzberg [1] modified a typical receiver to measure correlation power at these offset values of delay and Doppler using a nadir-pointing LHCP antenna.

In Fig. 2, we illustrate the type of swath coverage that GPS provides. Because of the multiplicity of GPS transmitters the receiver can observe a number of simultaneous footprints on the surface. This illustrates that while the system does not provide imaging in a standard sense (like a cross-scanning system, for example), it does provide more spatial coverage than from a sensor that is only able to view one ground point at a time.

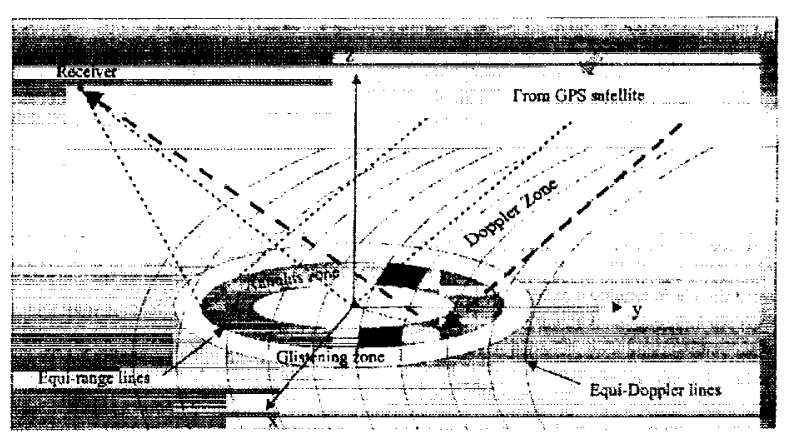

Figure 1. Illustration of glistening zone (adapted from Komjathy et al., 1999). 


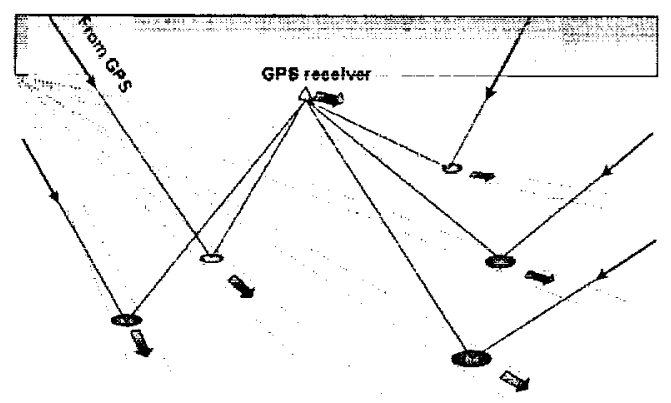

Figure 2. GPS swath coverage.

Results and observations suggest that the reflected GPS signal is very sensitive to the presence of sea ice, and furthermore, contains information related to the reflection coefficient of different ice conditions.

The reflection coefficient of a frozen sea surface is determined by the effective dielectric constant of ice and, under some conditions, by the dielectric constant of the underlying water. The latter is important for thin ice conditions where a significant portion of the radio wave energy may reach the second interface, between the ice and water. The effective dielectric constant of ice depends on various factors, such as an ice composition, salinity, temperature, density, age, origin, morphology, etc. [11]. Because first-year ice has needle-like inclusions of brine (predominantly oriented along the vertical direction), this variety of ice is notably anisotropic. Therefore, the dielectric constant is a tensor rather than a scalar constant. This makes the problem of modeling and interpreting the GPS signal scattering from ice rather challenging. At the same time, the sensitivity of the GPS scattered signal to these complex ice characteristics makes it a particularly attractive remote sensing tool.

Given the unique potential offered by reflected GPS signal sensing (e.g., L-band observations, forward-scattering sensing, low-cost and simple devices useable on any type of aircraft, etc.) and the geophysical significance of additional sea ice and permafrost data, research to investigate and exploit GPS returns from ice and frozen ground is warranted. However much remains to be done to determine the consistency of the signal return from ice surfaces; to investigate the information content of the signals; and to develop simplified retrieval algorithms to extract the cryospheric conditions.

\section{EXPERIMENTS AND DATA ANALYSIS}

Most of the GPS reflection experiments to date have been conducted with the Delay Mapping Receiver (DMR) designed by Drs. Katzberg and Garrison [1], based on the GEC Plessey (now MITEL) GPSBuilder-2 [12]. The DMR has two antenna inputs to allow standard tracking of direct signals using a zenith-pointed RICP antenna and correlation measurements of reflected signals using a downward-pointed LHCP antenna. DMR makes measurements of the correlation between the reflected signal and shifted versions of the local signal replica. This provides a trace of the reflected signal correlation as a function of code delay, thus mapping the return from annular regions of the glistening zone.

The first measurements of reflected GPS data from sea ice were collected by Dr. Maslanik in the Beaufort Sea in April 1998. During the experiment, the GPS system was tested with antennas mounted on a boom and held over various ice surfaces at different heights ranging from $30 \mathrm{~cm}$ to $20 \mathrm{~m}$. Each antenna was mounted on a small $20 \mathrm{~cm}$ by $20 \mathrm{~cm}$ aluminum ground plane, with the two plates then attached to provide the up-looking and down-looking mount for the antennas. A second reflected GPS data set was collected north of Barrow, Alaska in April 1999, using the same system but in this case mounted on a Cessna 185. Approximately 2 hours of data were collected in conjunction with a National Ice Center (NIC) reconnaissance flight and nearly coincident in time with a RADARSAT overpass.

Our experiment to observe GPS reflections from Arctic sea ice showed that for the moderate altitudes of the airbome GPS receiver, the reflected signal shape was fairly consistent (sharp, narrow waveform throughout the flight) indicating that ice surface roughness variations were not significant at L-band. On the other hand, the peak power was found to change significantly along the flight track. This behavior of the signal is a clear indication of the sensitivity to ice reflection coefficients. Correlations seen in our comparison between RADARSAT backscatter and GPS forward scattered data indicate that the GPS signal may indeed provide useful information regarding ice conditions, in addition to the basic ability to detect the presence of sea ice.

Fig. 3 shows the peak return power from GPS satellite PRN 30, collected during the flight. Reflected power is plotted versus RADARSAT backscatter along the flight leg, as a function of along track epochs. The two sets of curves indicate a positive correlation (see Labels 1 to 6 ) between the forward-scattered GPS peak power and the backscattared RADARSAT data for locations of high reflection coefficients, as well as some other variations in the GPS data that differ from the RADARSAT backscatter. Observation of positive rather than negative correlation requires further investigation.

In Fig. 4 we present a photo taken from the aircraft during the flight. The picture were taken when the aircraft flew by Label 5. The correspondence of the reflected peaks with the occurrence of large leads in the same area suggests that the high signal returns may have originated from patches of new or young ice as observed from the aircraft. In Fig. 4, we also indicated an illustration of the possible specular reflection track crossing a region of recently formed ice resulting in high GPS signal returns shown as gray line. Low signal 
returns around this region may have been due to thicker, first year ice conditions (dark line).

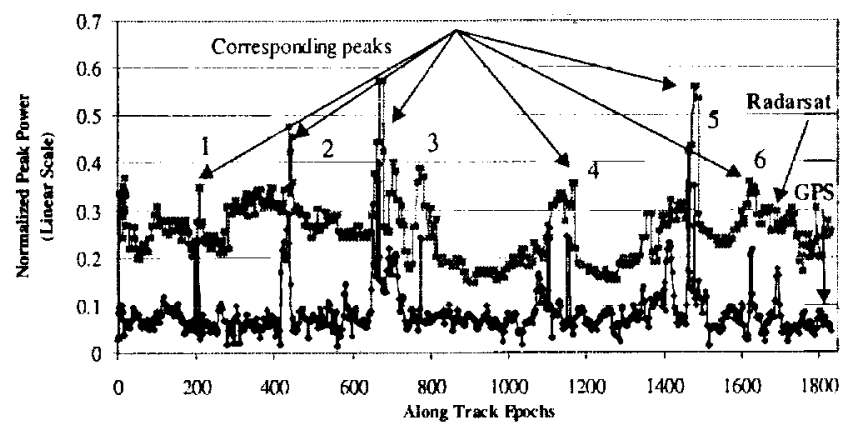

Figure 3. Normalized signal power for PRN 30.

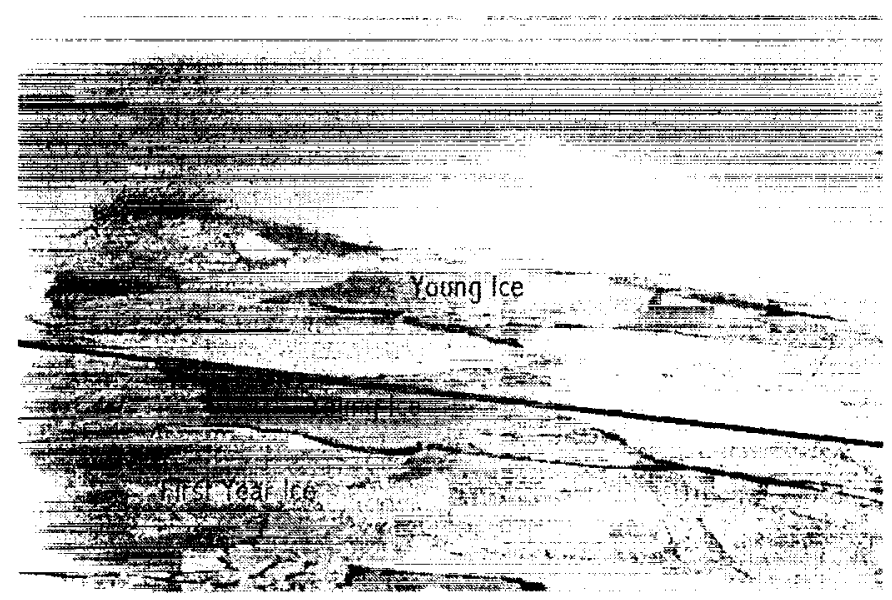

Figure 4. Photo taken from the aircraft in the vicinity of Label 5 showing black and darker gray areas of newly formed leads, new and young ice.

\section{CONCLUSIONS}

Field experiments, including data acquired from aircraft flights over the ice pack near Barrow, Alaska, suggest that the reflected GPS signals contain useful information over sea ice. Given this new potential application for GPS remote sensing, an effort to investigate reflected GPS signals over sea ice is discussed in the paper. A combination of modeling considerations, in-situ measurements and aircraft observations have been presented to quantify the theoretical and observed relationships between reflected GPS signals and cryospheric conditions.

\section{ACKNOWLEDGMENTS}

The research was funded by NASA Langley Research Center (LaRC) under grant no. NAG-1-1927. Thanks are due to A. Ulak of NIC for provision of ice reconnaissance information. RADARSAT data were obtained from NIC and the Alaska SAR Facility.

\section{REFERENCES}

[1] J.L. Garrison, S.J. Katzberg, and C.T. Howell, "Detection of ocean reflected GPS signals: theory and experiment," In the Proceedings of the IEEE Southeastcon 1997, Blacksburg, USA, pp. 290-294, 12-14 April 1997.

[2] J.L. Garrison, S.J. Katzberg, and M.I. Hill, "Effect of sea roughness on bistatically scattered range coded signals from the Global Positioning System," Geophys. Res. Letters, Vol. 25, No. 13, pp. 2257-2260, 1998.

[3] S.J. Katzberg and J.L Garrison "Utilizing GPS to determine ionospheric delay over the ocean," NASA Tech. Memo.- 4750, 1996.

[4] S.J Katzberg, J.L. Garrison, N.C. Coffey, and H.R. Kowitz, "Method and system for monitoring sea state using GPS," Application filed with the U.S. Patent and Trademark Office, 8 January, 1998.

[5] B. Lin, S.J. Katzberg, J.L. Garrison, and B. Wielicki, "The relationship between the GPS signals reflected from sea surface and the surface winds: Modeling results and comparisons with aircraft measurements," J. Geophys. Res., Vol., 104(C9), pp.20,713-20,727, 1999.

[6] S.F. Clifford, V.I. Tatarskii, A.G. Voronovich, and V.U. Zavorotny, "GPS sounding of ocean surface waves: theoretical assessment," In Proc. Int. Geosci. and Remote Sens. Symp. (IGARSS), Vol. IV, Seattle, USA, pp. 2005-2007, 1999.

[7] V.U Zavorotny and A.G. Voronovich, "Scattering of GPS signals from the ocean with wind remote sensing application," IEEE Trans Geosci. Remote Sens., Vol. 38, No. 2, pp.951-964, 2000.

[8] V.U. Zavorotny and A.G. Voronovich, "Bistatic radar scattering from an ocean surface in the small-slope approximation." in the Proceedings of the IEEE International Geoscience and Remote Sensing Symposium: Remote Sensing of the System Earth - A Challenge for the 21 st Century, 99CH36293, pp. 2419 2421, IEEE, Piscataway, N. J., 1999.

[9] A. Komjathy, V.U. Zavorotny, and J.L. Garrison, "GPS: a new tool for ocean science," GPS World, Vol. 10, No. 4, pp. 50-56, 1999.

[10] A. Komjathy, V.U. Zavorotny, P. Axelrad, G.H. Born and J.L. Garrison, "GPS signal scattering from sea surface: wind speed retrieval using experimental data and theoretical model," J. Remote Sens. Env., 2000 (in press).

[11] M.E. Shohr, "Field observations and model calculations of dielectric properties of Arctic sea ice in the microwave C-band," IEEE Trans. on Geosci and Remote Sens., Vol. 36, No. 2, pp.463-478, 1998.

[12] GEC Plessey Semiconductors, Global Positioning Products Handbook, 1996. 


\title{
A Comparison of GPS and Scatterometer Sensing of Ocean Wind Speed and Direction
}

\author{
Michael Armatys ${ }^{1}$, Attila Komjathy ${ }^{1}$, Penina Axelrad ${ }^{1}$, Stephen J. Katzberg ${ }^{2}$ \\ ${ }^{1}$ CCAR/University of Colorado, Campus Box 431, Boulder, CO 80309 \\ ${ }^{2}$ NASA Langley Research Center, Code 328, Hampton, VA 23681 \\ 303-492-3489 (P) / 303-492-2825 (F) / armatys@colorado.edu
}

\begin{abstract}
Initial estimates of ocean surface wind speed and direction based on observations of reflected GPS signals are presented and compared to QuikSCAT wind fields. The two wind speed estimates are generally in agreement to within $2-3 \mathrm{~m} / \mathrm{s}$, and under favorable conditions of well developed seas and stable winds, direction estimates agree to within $10 \mathrm{deg}$. An overview of the GPS technique is presented as well as a presentation and discussion of these first results.
\end{abstract}

\section{INTRODUCTION}

In 1996, NASA researchers Stephen J. Katzberg and James L. Garrison identified the potential application of the Global Positioning System (GPS) to remote sensing of ocean surface conditions $[1,2]$. Their concept was to use GPS in a bistatic radar configuration with the GPS satellite transmitting an L-Band spread spectrum signal, and the receiver on an aircraft or spacecraft platform measuring the reflected signal characteristics. Since then, Katzberg and Garrison have continued to investigate the properties of the ocean-reflected GPS signals and have developed a specialized Delay-Mapping GPS receiver (DMR) to measure the reflected signals $[1,3]$. Using the DMR and geophysical models to predict the interaction of the GPS signals with sea water, researchers have been able to estimate wind speeds on the ocean surface with an accuracy of $2 \mathrm{~m} / \mathrm{s}$ [1]. Recently we have developed a new approach that extends the GPS-based wind retrieval to determination of wind direction as well as wind speed, based on measurements from two or more reflected GPS signals [3]. To validate the performance of GPS for wind vector retrieval we have previously relied on buoy data. and wind speed estimates from TOPEX $\backslash P O S E I D O N$ [4].

This paper describes our first comparisons to wind vectors derived from the SeaWinds scatterometer onboard QuikSCAT. Ku-Band scatterometers represent the current state-of-the-art in sea surface wind remote sensing, providing highly accurate measurements with good temporal and spatial resolution on a global scale. The QuikSCAT science team provides a variety of wind products including wind vector maps at $25 \mathrm{~km}$ resolution. Nominal accuracy for the QuikSCAT wind vectors is 2 $\mathrm{m} / \mathrm{s}$ and $20 \mathrm{deg}$ for wind speed and direction, respectively. Performance is somewhat worse for wind speeds below 3 $\mathrm{m} / \mathrm{s}$, for grid points within $25 \mathrm{~km}$ of land, and for points very close to nadir or at large look angles. Algorithms are included in the QuikSCAT data reduction that resolve wind direction ambiguities and corresponding wind speed estimates. QuikSCAT observations provide a snapshot of the ocean surface conditions at the time of the satellite overflight. The backscatter measurements at Ku-Band are sensitive to rapid changes in the surface conditions making this a powerful sensor for wind retrieval. Because of the substantially different mechanisms for forward scattered L-Band reflections, the measurement and retrieval of wind vectors from GPS may provide a very useful complement to scatterometer observations in improving global wind observations and models.

\section{GPS-BASED WIND ESTIMATION}

As presented in [1, 2, 3], GPS-based wind retrievals rely on the modification of the correlation function for signals reflected by rough surfaces. The basic observable is not the pseudorange or carrier phase measurement used for most GPS applications, but rather a measure of the correlation of the reflected signal with a delayed replica of the PRN code. The distribution of this function over delays (and Dopplers) provides a mapping of the reflecting surface. In particular we use a model developed by $\mathrm{Za}$ vorotny and Voronovich $(\mathrm{Z}-\mathrm{V})[5,6]$ for prediction of the reflected signal structure. The Z-V model embodies the forward-scatter radar equation with the geometric optics limit of the Kirchhoff Approximation. The distribution of ocean surface slopes is assumed to be Gaussian with variances determined by a wave spectra model such as that developed by Elfouhaily et al. [7]. These slope statistics define the shape and orientation of the glistening zone over which GPS signals are reflected toward the receiving antenna. The models currently used predict the maximum variance in the up/down-wind direction and minimum variance in the cross-wind direction. This allows for the identification of wind direction with a $180 \mathrm{deg}$ ambiguity. More advanced models that include up/downwind asymmetry due to effects such as Bragg scattering are currently under development.

To estimate wind vectors from GPS observations we first determine the surface slope statistics in the model which best fit the data. The second step is to use an ocean model to retrieve a wind speed and direction estimate from the slopes. Two satellites are necessary to recover the directional information lost in measuring the return from the entire annulus of the glistening zone with the DMR. In the current implementation, we try to use satellites with relatively high elevation angles to insure that the specular points are nearly co-located and that the elevation 
angle-dependent slope statistics are nearly the same for both satellites. A degenerate case occurs when the angle between the incident planes of the two GPS satellites is very close to $90 \mathrm{deg}$. In this situation only the magnitude of the angle between the incident plane and the up/downwind direction can be determined, and an additional ambiguity exists in the wind direction.

The correct wind speed is chosen by interpolating the estimated slope statistics against the slope statistics from the Elfouhaily model [7]. Underlying the wave spectra and Z-V models are assumptions about the ocean conditions that affect the performance expected. In particular, the current model assumes well developed seas, with no swell. Coastal effects are not included. These simplifying assumptions limit the applicability of the current implementation under coastal and highly variable ocean conditions.

\section{INITIAL RESULTS}

On February 28 and March 11, 2000, Dr. Katzberg conducted two flight tests in a B200 aircraft off the Virgina coast to gather GPS reflection data coincident with QuikSCAT overflights. Fig. 1 and Fig. 3 show the QuikSCAT wind fields and aircraft flight tracks for March 11 and February 28, respectively. The February 28 map also shows the location of three buoys for which wind speed and direction data was logged by the National Data Buoy Center (NDBC). (Unfortunately, similar buoy data for March 11, 2000 has not been archived.) DMR data logged at $10 \mathrm{~Hz}$ were grouped into one-minute segments numbered sequentially along the flight tracks. Results of the GPS-based wind vector retrievals for each one minute sample on the two days are shown in Figs. 2 and 4.

The March 11 QuikSCAT wind field shows very stable winds in the range from $8.5-10 \mathrm{~m} / \mathrm{s}$, due north (within $+/$ $10 \mathrm{deg}$ ) over the entire aircraft flight path. GPS-derived wind direction estimates, shown in Fig. 2b are also consistently within about $5 \mathrm{deg}$ of North. GPS-based wind speeds for the first 10 samples are quite low, reporting values as low as $5 \mathrm{~m} / \mathrm{s}$ as compared to the consistent $10 \mathrm{~m} / \mathrm{s}$ QuikSCAT result. Beginning at sample 18, the GPS wind speed estimates move toward the QuikSCAT values with most of the points falling within the $2 \mathrm{~m} / \mathrm{s}$ error range.

This data set presents a very favorable situation for GPS versus QuikSCAT comparisons. The wind field is quite stable and does not originate over the land; thus the wave spectra model assumptions should be valid under these conditions and good agreement is to be expected. The discrepancy in wind speed may be related to a relatively large difference between the elevations of the two satellites at the beginning of the flight. Initially one satellite is at an elevation of $44 \mathrm{deg}$ while the second is at $63 \mathrm{deg}$. By the middle of the flight, the first has risen to $53 \mathrm{deg}$. We expect that a more accurate wind speed estimate can be obtained using only one of the two satellites or by modifying our algorithm to accommodate different elevations.

The February 28 QuikSCAT wind field presented in Fig. 3 shows quite a different situation with relatively low wind speeds ( $<6 \mathrm{~m} / \mathrm{s}$ ) throughout, and wind directions that vary over 70 deg. Furthermore, winds along the flight path originate over the land areas. Surprisingly, GPS wind speed estimates fall within $2 \mathrm{~m} / \mathrm{s}$ of the QuikSCAT estimates, despite the low wind speeds and a large difference in satellite elevations. The GPS-derived wind direction is very stable, in the range of $80-90 \mathrm{deg}$ throughout the flight, not at all reflecting the rapid variations seen in the QuikSCAT estimates. There are number of possible explanations for this, most important of which is the low wind speed, which may lead to unreliable direction estimates for both methods. A second possibility is the longer averaging time inherent in the GPS L-Band observations and data grouping. Hourly data from all three buoys shows consistent wind direction in the range of 90 $110 \mathrm{deg}$ over a two hour period surrounding the flight tests. A third consideration is related to the GPS satellite geometry. Throughout this fight, the azimuthal separation of the two GPS satellites was very close to 90 deg. As described previously, this degenerate case leads to an ambiguity in the wind direction related to the angle between the wind and the incident plane. For a true wind direction of $90 \mathrm{deg}$, at the start of the experiment (Sample 1), the ambiguity is such that the possible wind directions are only $90 \mathrm{deg}$ and $270 \mathrm{deg}$; however, for sample 30 , the two satellite azimuths have shifted by $30 \mathrm{deg}$, resulting in possible wind directions of $90,150,270$, and $330 \mathrm{deg}$.

\section{CONCLUSIONS}

The results presented here represent the first comparisons between GPS-derived ocean surface wind vectors and QuikSCAT wind fields. We plan to continue these comparisons with additional flight experiments and anal$y$ sis under both favorable and challenging conditions with the goal of exploring the possibility of using GPS as a complimentary sensor to airborne and spaceborne scatterometers. The use of GPS for wind retrievals, is an emerging technology with considerable opportunity for further development of the theory and instrumentation, and significant potential for contributing to our understanding of regional and global ocean surface conditions.

\section{ACKNOWLEDGMENTS}

The authors would like to thank Dr. Zavorotny for his technical discussions on forward scatter signal models. We would also like to thank Dr. Elfouhaily for his insight into wind direction ambiguity resolution. The research was funded by NASA Langley Research Center (LaRC) under grant no. NAG-1-1927.

\section{REFERENCES}

[1] J. L. Garrison, S. J. Katzberg, and M. I. Hill. "Effect of Sea Roughness on Bistatically Scattered Range Coded Signals from the Global Positioning System". Geophysical Resarch Letters, 25:2257-2260, 1998.

[2] J. L. Garrison, S. J. Katzberg, and C. T. Howell, III. "Detection of Ocean Reflected GPS Signals: Theory 


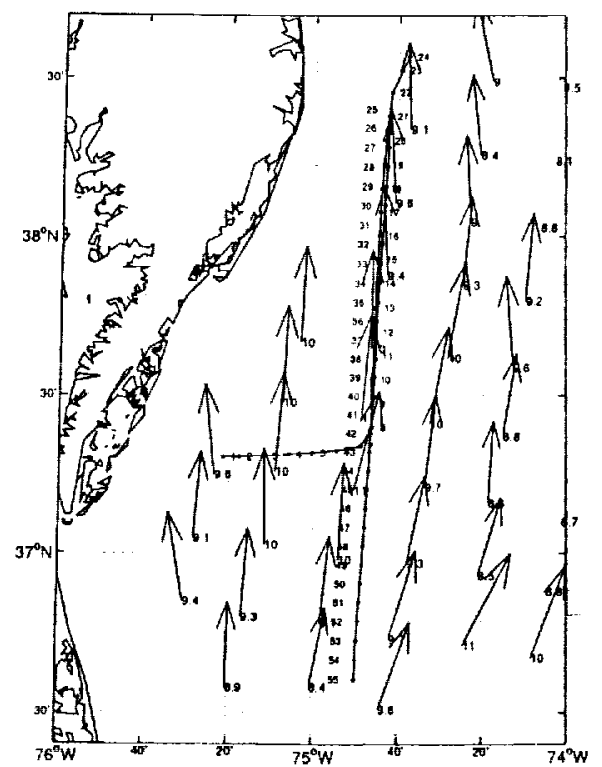

Figure 1: Quiver plot of QuikSCAT data for 11 March, 2000. Also shown is the aircraft flight path.

and Experiment". In Proceedings of the IEEE Southeastcon '97: Engineering the New Century, pages 290294, Blacksburg, VA, 1997.

[3] M. Armatys, D. Masters, A. Komjathy, P. Axelrad, and J. L. Garrison. "Exploiting GPS as New Oceanographic Remote Sensing Tool". In Proceedings of the 2000 National Technical Meeting of the Institute of Navigation, Anaheim, CA, 26-28 Jan 2000. In press.

[4] A. Komjathy, V.. Zavorotny, P. Axelrad, G. Born, and J. Garrison. "GPS Signal Scattering from Sea Surface: Wind Speed Retrieval Using Experimental Data and Theoretical Model". Journal of Remote Sensing of Envrironment, 2000. In press.

[5] S. F. Clifford, V. I. Tatarskii, A. G. Voronovich, and V. U. Zavorotny. "GPS Sounding of Ocean Surface Waves: Theoretical Assessment". In Proceedings of the IEEE International Geoscience and Remote Sensing Symposium: Sensing and Managing the Environment, pages 2005-2007, Piscataway, NJ, 1998.

[6] V. U. Zavorotny and A. G. Voronovich. "Scattering of GPS Signals From the Ocean With Wind Remote Sensing Application". IEEE Transactions on Geoscience and Remote Sensing, 38(2):951-964, Mar 2000.

[7] T. Elfouhaily, B. Chapron, K. Katsaros, and D. Vandemark. "A Unified Directional Spectrum for Long and Short Wind-Driven Waves". Journal of Geophysical Research, 102(C7):15781-15796, 15 Jul 1997.
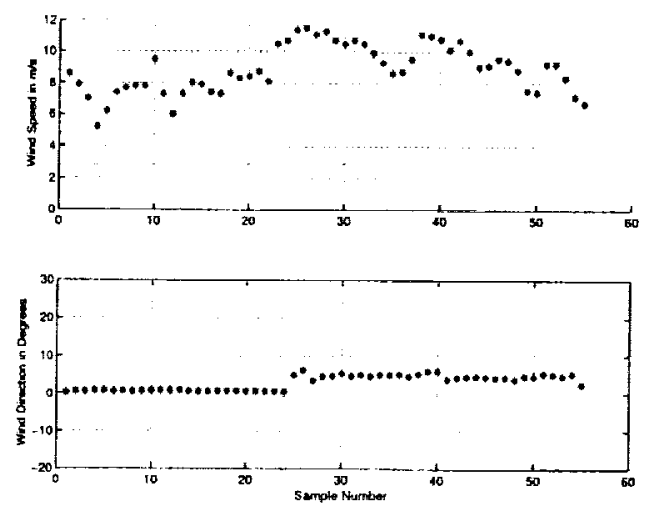

Figure 2: Wind speed (a) and wind direction (b) estimates from GPS observations for $11 \mathrm{March}, 2000$ GPS data.

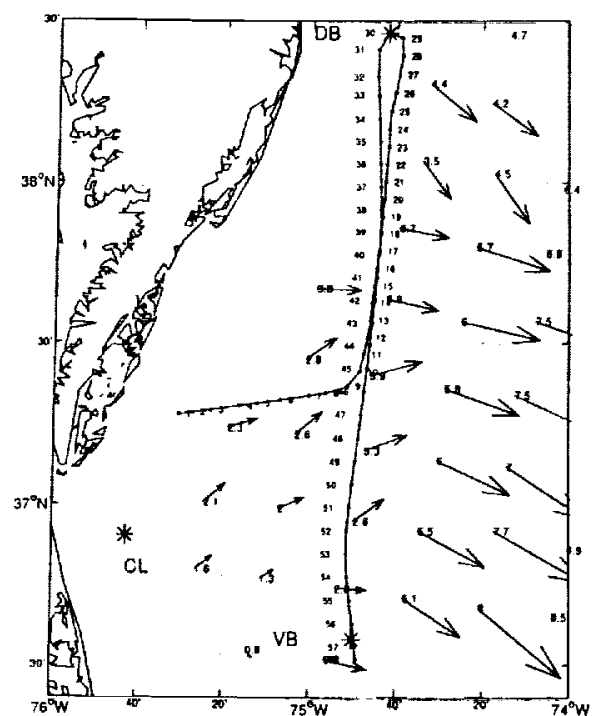

Figure 3: Quiver plot of QuikSCAT data for 28 February, 2000. Also shown are the aircraft flight path, and the locations of the Delaware Bay (DB), Chesapeake Light $(\mathrm{CL})$, and Virginia Beach (VB) buoys.
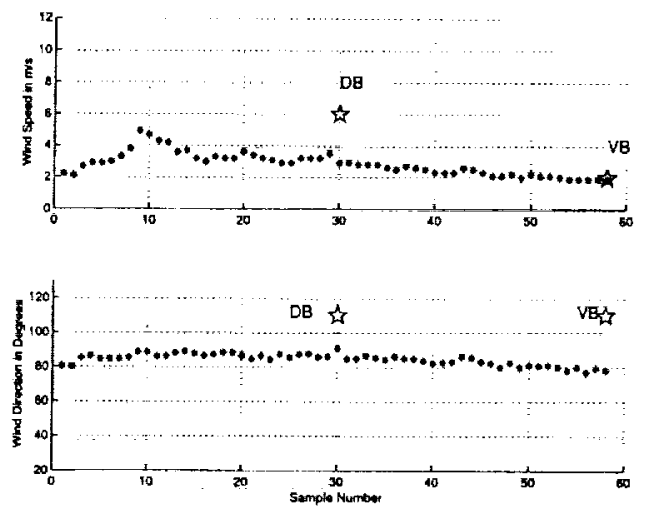

Figure 4: Wind speed (a) and wind direction (b) estimates from GPS observations for 28 February, 2000 GPS data. Also shown on the plots are the Delaware Bay (DB) and Virginia Beach (VB) buoy estimates at the positions in the flight track closest to the buoy positions. 